\title{
Existing Motor State Is Favored at the Expense of New Movement during 13-35 Hz Oscillatory Synchrony in the Human Corticospinal System
}

\author{
Thomas Gilbertson, ${ }^{1}$ Elodie Lalo, ${ }^{1,2}$ Louise Doyle, ${ }^{1}$ Vincenzo Di Lazzaro, ${ }^{3}$ Beatrice Cioni, ${ }^{4}$ and Peter Brown ${ }^{1}$ \\ ${ }^{1}$ Sobell Department of Motor Neuroscience and Movement Disorders, Institute of Neurology, London WC1N 3BG, United Kingdom, ${ }^{2}$ Equipe d'accueil en \\ Sciences du Sport, Université Marc Bloch, de Strasbourg II, F67084, France, and Institutes of ${ }^{3}$ Neurology and ${ }^{4}$ Neurosurgery, Università Cattolica, 00168 \\ Rome, Italy
}

\begin{abstract}
Oscillations in local field potentials in the $\beta$-frequency band $(13-35 \mathrm{~Hz})$ are a pervasive feature of human and nonhuman primate motor cortical areas. However, the function of such synchronous activity across populations of neurons remains unknown. Here, we test the hypothesis that $\beta$ activity may promote existing motor set and posture while compromising processing related to new movements. Three experiments were performed. First, healthy subjects were instructed to make reaction time movements of the outstretched index finger in response to imperative cues triggered by transient increases in corticospinal synchrony, as evidenced by phasic elevations of $\beta$-frequency band microtremor and intermuscular synchrony. Second, healthy subjects were instructed to resist a stretch to the index finger triggered in the same way. Finger acceleration in the reaction time task and transcortical components of the stretch reflex were measured and compared with those elicited by random cue or stretch presentation. Finally, we sought a correlation between finger acceleration in the reaction time task and cortical synchrony directly measured from the electrocorticogram in two patients undergoing functional neurosurgery. We demonstrate that movements are slowed and transcortical responses to stretch are potentiated during periods of elevated $\beta$-band cortical synchrony. The results suggest that physiological periods of $\beta$ synchrony are associated with a cortical state in which postural set is reinforced, but the speed of new movements impaired. The findings are of relevance to Parkinson's disease, in which subcortical and cortical $\beta$-band synchronization is exaggerated in the setting of increased tone and slowed movements.
\end{abstract}

Key words: sensorimotor cortex; oscillatory synchrony; corticospinal system; motor set; transcortical reflexes; voluntary movement

\section{Introduction}

Synchronized oscillations across populations of neurons are a pervasive feature of brain function. Most investigations have focused on their role in the sensory domain, but oscillations in local field potentials (LFPs) are also apparent at multiple levels of the human and nonhuman primate motor system. In particular, rhythmic activity in the $\beta$-frequency band (13-35 Hz) can be recorded from the motor cortex (Murthy and Fetz, 1992; Sanes and Donoghue, 1993; Baker et al., 1997), cerebellar system (Marsden et al., 2000; Paradiso et al., 2004), and basal ganglia (Brown et al., 2001; Levy et al., 2002; Williams et al., 2002; Silberstein et al., 2003; Courtemanche et al., 2004), and its consequence is readily picked up in the periphery through the synchronization of motor units within and between muscles (Farmer et al., 1993; Conway et al., 1995; Gibbs et al., 1995; Baker et al., 1997; Salenius et al., 1997; Kilner et al., 1999) and in the mechanical product of such synchronization, in the form of microtremor (McAuley et al., 1997; Halliday et al., 1999).

\footnotetext{
Received May 3, 2005; revised June 27, 2005; accepted July 15, 2005.

This work was supported by the Amulree Trust (T.G.), Association France-Parkinson (E.L.), and the Medical Research Council (London, UK) (L.D. and P.B.).

Correspondence should be addressed to Peter Brown at the above address. E-mail: p.brown@ion.ucl.ac.uk. DOI:10.1523/JNEUROSCI.1762-05.2005

Copyright $\odot 2005$ Society for Neuroscience $\quad$ 0270-6474/05/257771-09\$15.00/0
}

LFP oscillations in the $\beta$ band arise through the synchronization of populations of local neurons, so that the discharges of single neurons are locked to the LFP oscillations at different levels of the motor system (Murthy and Fetz, 1992, 1996; Sanes and Donoghue, 1993; Baker et al., 1997, 2003; Donoghue et al., 1998; Levy et al., 2002; Courtemanche et al., 2004; Kühn et al., 2005). However, the function of such synchronization within the motor system remains unknown, although some inverse relationship with the processing of new movements seems likely given the consistent suppression of $\beta$-band LFP activity by movement (Sanes and Donoghue, 1993; Donoghue et al., 1998; Kilner et al., 1999; Cassidy et al., 2002; Paradiso et al., 2004). Moreover, subcortical and cortical activity in the $\beta$-frequency band is pathologically increased in Parkinson's disease (Brown et al., 2001; Silberstein et al., 2003, 2005) and in nonhuman models of parkinsonism (Sharott et al., 2005), states dominated by deficient movement and rigidity. In patients with Parkinson's disease, slow movements are correlated with delayed and deficient suppression of $\beta$-band LFP oscillations in the subthalamic nucleus (Kühn et al., 2004; Williams et al., 2005), whereas the voluntary inhibition of movement is associated with a relative increase in $\beta$ activity in this structure (Kühn et al., 2004).

Together, these observations suggest that $\beta$ activity may promote the existing motor set and any accompanying postural con- 
traction while compromising local motor processing related to new movements. Here, we test this hypothesis with respect to synchronization of populations of neurons in cerebral cortical motor areas in healthy subjects. The predictions are twofold: movements "triggered" during periods of elevated $\beta$-frequency band synchrony will be slowed, whereas transcortical stretch reflexes that reinforce existing posture will be potentiated.

\section{Materials and Methods}

Subjects. Ten right-handed healthy subjects (mean age, 29 years; range, 22-35 years; two females) participated in the reaction time (RT) paradigm and 10 right-handed healthy subjects (mean age, 32 years; range, 22-44 years; all males) in the finger stretch reflex paradigm. In addition, in two patients who had received functional neurosurgery for treatment of chronic pain, we recorded from epidural electrode strips implanted over the sensorimotor cortex. The electrode strips (Resume; Medtronic, Minneapolis, MN) consisted of four contacts $(0-3)$ of $4 \mathrm{~mm}$ in diameter, each separated by $1 \mathrm{~cm}$. In Patient DF, a 57-year-old male with a 7 year history of multiple sclerosis and right-sided trigeminal neuralgia, a single strip was implanted across the central sulcus. The N20 component of the cortical potential evoked by intraoperative electrical stimulation of the contralateral median nerve at the wrist underwent polarity reversal at contact 1 . This, together with the low-threshold motor response to intraoperative direct electrical stimulation, confirmed that the most anterior contacts, 2 and 3, overlay the motor cortex. Patient DF was recorded $6 \mathrm{~d}$ postoperatively while off medication. In subject CO, a 54-year-old female with a 3 month history of left-sided upper limb pain after basal ganglia stroke, two electrode strips were implanted parallel to and on either side of the right central sulcus. In the posterior electrode strip, the N20 response was largest over contacts 1 and 2, whereas stimulation over contacts 0 and 1 of the anterior electrode strip elicited the largest motorevoked potential in abductor pollicis brevis. Patient $\mathrm{CO}$ was recorded $1 \mathrm{~d}$ postoperatively while on medication (daily dosage: $1 \mathrm{mg}$ of clonazepam, $800 \mathrm{mg}$ of gabapentin, and $300 \mathrm{mg}$ of Ox-carbamazepine). All subjects participated with informed consent and the permission of the local ethics committees.

Paradigms and recordings. The first prediction we sought to test was that movements triggered during periods of elevated $\beta$-frequency band synchrony among relevant cortical neurons would be slowed in healthy subjects. The most direct way to determine such synchronization in real time would be through time-evolving estimates of spectral power in direct records of electrocorticographic (ECoG) signals because these have excellent spatial resolution and signal-to-noise ratios. However, this was not possible in healthy subjects, because time-evolving estimates of spectral power in scalp recordings of electroencephalographic (EEG) or magnetoencephalographic (MEG) activity had insufficient spatial resolution and signal-to-noise ratios for our purposes. The spatial resolution with which synchronization can be detected among cortical neurons active in a given task can be considerably improved by estimating the coherence between EEG or MEG signals and muscle activity. However, this approach has poorer temporal resolution and, more importantly, entails delayed estimates of synchrony through computational demands and the need to estimate coherence over data blocks. Thus, in this study, we elected to use the mechanical product of synchronized cortical activity, finger microtremor in the $\beta$ band, as our primary, albeit indirect, time-evolving index of the synchronization between active cortical neurons (McAuley et al., 1997; Halliday et al., 1999). We developed a simple visually cued reaction time paradigm in which the time of the presentation of the cue was determined by the stochastic incidence of a microtremor oscillation. Subjects were instructed to abduct their extended right index finger $\geq 10^{\circ}$ as quickly as possible on illumination of a lightemitting diode, and the speeds of these triggered movements were compared with those performed when the same cue was presented at random time intervals during the same recording session. Both triggered cues (TCs) and random cues (RCs) were delivered at a minimum interval of $5 \mathrm{~s}$ with RC presentation determined by a pseudorandomized output device (D4030 pulse generator; Digitimer, Welwyn Garden City, UK) with a maximum interval of $30 \mathrm{~s}$.
Subjects were seated with their forearms supported on a table with both their right and left index fingers abducted slightly and tonically extended against gravity. Finger microtremor was detected by accelerometers (EGAXT-50; Entran, Fairfield, NJ) attached to the medial aspect of the distal phalanx of the right and left index finger. The microtremor signal was amplified and filtered on-line using a custom-built 16th-order elliptic 13-35 Hz bandpass electronic filter (stopband attenuation of 60 $\mathrm{dB}$ ) with an output delay of $50 \mathrm{~ms}$. After on-line rectification, the signal was used to generate a single triggering pulse when it surpassed a voltage threshold defined during a 30-60 s calibration period before the recording of each run and equivalent to two to three times the root mean square amplitude of the bandpass-filtered signal. Thus, the TC was presented 50 $\mathrm{ms}$ after the onset of phasic increases in $\beta$-frequency band microtremor. The threshold was adjusted to allow an approximately equal number of TCs to RCs during each session. Four or five recording sessions of 2-4 min runs, separated by $1-2 \mathrm{~min}$ rest, were performed over a $30 \mathrm{~min}$ period after a practice session of 3-5 min.

The second prediction of this study was that transcortical reflexes that reinforce posture should be enhanced during periods of oscillatory activity in the $13-35 \mathrm{~Hz}$ band. To test this prediction, we used the same microtremor burst-detection procedure outlined above but simply substituted the light-emitting diode for a sudden tangential force applied to the extended index finger. Triggered stretches (TSs) or random stretches (RSs) were delivered by a servo-controlled torque motor and consisted of sudden $1 \mathrm{~N}$ increases in tangential force delivered over $200 \mathrm{~ms}$. Subjects were asked to resist the adducting pull of the motor on the distal phalanx of the right index finger, so as to keep the finger in the same position. An elastic band linked the index finger to the lever of the torque motor, so that the microtremor of the finger was not dampened by inertial load (McAuley et al., 1997). Two experimental sessions were recorded on different days; in the first, the only delay between triggering and stretch presentation was the $50 \mathrm{~ms}$ output delay imposed by the on-line electronic $\beta$ filter. In the second session, there was an additional delay of 200 $\mathrm{ms}$, making a total delay of $250 \mathrm{~ms}$. For each session, TS and RS appeared in random order and approximately balanced number at intervals of $5-30$ s over a period of $\sim 15 \mathrm{~min}$. This was repeated twice, separated by a rest period of $2-3 \mathrm{~min}$.

We were fortunate enough to also record ECoG signals in two patients, so as to confirm that $\beta$ synchrony would be linked to motor slowing in direct recordings of cortical activity, albeit in patients with neurological disease. The reaction time paradigm was adapted so that only RCs were delivered in the patient recordings, in whom a correlation between randomly sampled spontaneous variations in $\beta$-band ECoG power and movement acceleration was sought across trials rather than a difference between sets of trials with two different levels of $\beta$-band microtremor. Patient $\mathrm{CO}$ was unable to produce comfortable finger abduction and thus made a brisk extension, rather than abduction, of their index finger on presentation of the cue.

In addition, electromyograms (EMGs) were recorded from the first dorsal interosseous (FDI) and extensor indicis (EI) using 9-mm silversilver chloride electrodes in a bipolar montage. EMG signals were amplified and bandpass filtered at $16-300 \mathrm{~Hz}$. Accelerometer signals were amplified and low-pass filtered at $100 \mathrm{~Hz}$ with no high-pass filtering. In the patients, ECoG was recorded in addition to acceleration. In patient $\mathrm{DF}$, the four electrode contacts of the epidural strip were configured to give three bipolar LFP signals, whereas in patient CO two epidural strips, each consisting of four contacts were configured in an identical montage to give six LFP signals. These were amplified and bandpass filtered between 0.9 and $250 \mathrm{~Hz}$. All signals were digitized with 12-bit resolution by a 1401 analog-to-digital converter (Cambridge Electronic Design, Cambridge, UK) and sampled at $1000 \mathrm{~Hz}$ using Spike 5.5 software (Cambridge Electronic Design) running on a personal computer.

Finally, in an ancillary experiment aimed at investigating the possible contribution of peripheral effects, stimulation (Stimulator Type 3073; Devices, Welwyn Garden City, UK) was delivered through two 9-mm silver-silver chloride surface electrodes, one fixed over the motor point of FDI and one fixed over the proximal interphalangeal joint of the tonically extended index finger. Shocks were square wave pulses of $0.2 \mathrm{~ms}$ duration of sufficient intensity to elicit twitches causing peak accelera- 
tions (PAs) of the index finger that approximately matched those seen in the reaction time experiments. Shocks were triggered by $\beta$ microtremor or delivered randomly as above, although an additional $285 \mathrm{~ms}$ delay was added to the timings of each shock so that they were delivered at a time that corresponded to the average reaction time in the standard cued paradigm.

Analysis in the reaction time paradigm. The extraction of the movement variables, RT and PA, was implemented off-line in Spike. RT was determined by measuring the time between cue onset and the start of the acceleration accompanying movement. PA was defined as the maximal acceleration in the direction of the voluntary movement within $100 \mathrm{~ms}$ of the initial acceleration point that defined the RT. This window was sufficient to capture the major deflection in the accelerometer trace. Any trials with an $\mathrm{RT}$ or triggering acceleration out with $3 \times \mathrm{SD}$ of the respective mean were rejected to limit artifacts because of very long reaction times and small shifts in the position of the finger, which nevertheless were associated with large accelerations leading to spurious triggering. Trials in which such a shift triggered the cue were distinguishable from genuine microtremor bursts, because the former consisted of abrupt nonoscillatory deflections in the raw acceleration trace that were considerably larger in amplitude than the background activity. An average of $89 \pm 7 \mathrm{TC}$ and $57 \pm 4 \mathrm{RC}$ trials were analyzed in the 10 subjects after a median of $10 \%$ of trials were rejected. In our ancillary experiment in which muscle twitches were elicited by direct electrical stimulation, the PA was measured in an average of $86 \pm 6$ triggered and $63 \pm 7$ random shocks in each of the 10 healthy subjects.

Finger tremor in the $\beta$-frequency band has been considered to be the mechanical product of synchronization of motor unit activity within and between muscles, which itself is attributable to synchronization within the $\beta$ activity in the corticospinal system (McAuley et al., 1997; Halliday et al., 1999). To confirm that periods of elevated $\beta$-band microtremor were accompanied by increased descending drive at similar frequencies in our paradigm, we derived a measure of short-term synchronization between the two muscles involved in the task, FDI and EI, using a wavelet-based approach. The Morlet wavelet function $\psi_{0}(\eta)$ is defined in nondimensional time $\eta$, where $\omega_{0}=6$ to localize the function in both time and frequency, and $i=\sqrt{ }-1$.

The following function provides a suitable compromise in both time and frequency to extract features from both EEG and EMG signals (Samar et al., 1999; Strambi et al., 2004):

$$
\psi_{0}(\eta)=\pi^{-1 / 4} e^{i \omega_{0} \eta} e^{-\eta^{2 / 2}} .
$$

The variations in amplitude of any signal at any frequency can be extracted from a time series $x_{n}$ by varying the wavelet scale $s$ and shifting along in time $n$ using the continuous wavelet transform (CWT):

$$
W_{n}(s)=\sum_{n^{\prime}=0}^{N-1} x_{n^{\prime}} \psi^{\star}\left[\frac{\left(n^{\prime}-n\right)}{s}\right],
$$

where the ${ }^{\star}$ indicates complex conjugation and $N$ is the number of points in the time series. All coefficients were normalized across all scales and derived in Fourier space to increase the speed of computation (Torrance and Compo, 1998). A value for intermuscular synchrony was derived by cross-correlating the wavelet decomposed EMG activity at the peak trigger frequency (PTF) during the triggered trials. This frequency was found after averaging the CWT of the accelerometer signal $0.4 \mathrm{~s}$ before the cue and $0.1 \mathrm{~s}$ after cue presentation. Wavelet coefficients were extracted at a time resolution of $1 \mathrm{~ms}$ and at scales corresponding to frequencies between 11 and $39 \mathrm{~Hz}$, with a resolution of $0.4 \mathrm{~Hz}$. To prevent amplitude cancellation attributable to averaging over trials, with phase differences relative to the trigger stimulus, all coefficients were converted to wavelet power. The CWT of the two EMG signals at the PTF were crosscorrelated over a $0.4 \mathrm{~s}$ precue and $0.1 \mathrm{~s}$ postcue period using a $128 \mathrm{~ms}$ overlapping sliding window shifted by $1 \mathrm{~ms}$. The cross-correlation coefficients were then averaged across the random or the triggered trials, and a peak value was extracted from each average within a window defined between $50 \mathrm{~ms}$ before the cue (allowing for the filter delay) and one cycle of the PTF. All values were normalized using Fisher's transform.
To establish whether fluctuations in ECoG oscillatory activity covaried with PA on a trial-to-trial basis the CWT of the LFP was derived for each millisecond over a $1.5 \mathrm{~s}$ period around the cue presentation. In patient $\mathrm{DF}$, the analysis window included $0.25 \mathrm{~s}$ post cue; however, in patient $\mathrm{CO}$, this period was extended to 0.4 because of a longer average reaction time. We chose the simplest model, a linear model, to describe the effect of $\beta$ activity after response acceleration. The base 10 logarithm was used to normalize the wavelet power across trials before calculating Pearson's correlation coefficient for the wavelet power at each time point and at each wavelet scale with the PA in that trial. The frequency at which the wavelet power correlated with the PA maximally was defined as the peak correlation frequency (PCF).

To determine the duration of bursts of $\beta$-frequency band microtremor, the wavelet power at the PTF was averaged around each time point at which power first exceeded the $95 \%$ confidence limit (CL) of background power. A similar approach was applied to the ECoG recorded from the patient. In this case, the PCF was used instead of the PTF.

Analysis in the triggered stretch paradigm. Any trials with a triggering microtremor $>3 \mathrm{SD}$ above the mean background $\beta$-band microtremor power were rejected to limit the effect of small shifts in the position of the finger. An average of $70 \pm 5$ TSs and $41 \pm 2$ RSs were analyzed in each subject for each delay between trigger and stretch after a median of $10 \%$ of trials were rejected. Full wave-rectified EMG signals in these trials were segmented into $1 \mathrm{~s}$ windows starting $500 \mathrm{~ms}$ before stretch onset. Windows were averaged separately with respect to RS and TS in each subject. The sizes of the short-latency spinal stretch-reflex response ("M1"), long-latency transcortical stretch-reflex response ("M2"), and after late response ("M3") components were defined as the mean amplitude of the total rectified EMG signals measured between 32 and $50 \mathrm{~ms}$ (M1), between 54 and $100 \mathrm{~ms}$ (M2), and between 100 and $300 \mathrm{~ms}$ (M3) after stretch onset (Marsden et al., 1983; Day et al., 1991; Macefield et al., 1996). Because the size of the long-latency response may vary with the level of background muscle contraction (Marsden et al., 1983), the mean area of the EMG activity measured for the $400 \mathrm{~ms}$ previous stretch onset was subtracted from the values obtained for M1, M2, and M3. The ratios between $\mathrm{M} 2$ responses measured after TS and RS were also calculated. An M2 TS/RS ratio $>1$ indicated a greater M2 response after TS compared with RS.

\section{Results}

Ten healthy subjects held their index finger tonically extended and were instructed to abduct this finger as quickly as possible in response to a visual cue, or, in a separate experimental session, to resist adducting stretches to the finger imposed by a torque motor. The acceleration signal from the tonically extended index finger revealed phasic bursts of microtremor in the $\beta$-frequency $(13-35 \mathrm{~Hz})$ band between reaction time movements or stretches. Such microtremor bursts were associated with a series of simultaneous EMG discharges in the FDI and EI muscles repeating at intervals of $\sim 50 \mathrm{~ms}$ (Fig. 1) and were used to trigger visual cues or finger stretches after a $50 \mathrm{~ms}$ delay imposed by the use of an on-line electronic $\beta$-frequency band triggering device. Note that microtremor bursts were prolonged (Fig. $1 A$ ) and continued beyond the triggering oscillation in triggered trials (Fig. 1D). Across the 10 healthy subjects, microtremor bursts exceeding the $95 \%$ $\mathrm{CL}$ of the mean power in the $\beta$-frequency band occurred every $2.7 \pm 0.3 \mathrm{~s}$, exceeding this level for $1.65 \pm 0.03$ cycles of the PTF, with a mean maximum duration of $3.4 \pm 0.26$ PTF cycles. Because bursts only exceeded 95\% CL of the mean power near their peaks, these represent conservative estimates of microtremor burst duration.

\section{Differences between trials in triggered cue experiments}

Correct triggering from periods of high $\beta$-band microtremor in TC trials was confirmed by averaging the acceleration wavelet power around the cue period in each subject (Fig. $2 A, B$ ). The 
mean PTF across subjects was $25 \pm 0.9 \mathrm{~Hz}$. The hypothesis that periods of high $\beta$-band microtremor were attributable to phasic increases in corticospinal synchrony in the $\beta$-frequency band was supported by a simultaneously elevated intermuscular synchrony at the PTF in TC, but not RC, trials (Fig. 2C-F). This phasic increase in intermuscular synchrony at the time of triggering in TC trials was unilateral and involved periods of $\sim 50 \mathrm{~ms}$, consistent with synchronization between motor units at $\sim 20 \mathrm{~Hz}$ (Fig. 2C). Overall, the peak cross-correlation coefficient was higher in TC compared with RC trials across subjects $(0.25 \pm 0.05$ and $0.13 \pm$ 0.02 ; Wilcoxon signed rank test; $Z=1.98$; $p=0.04)($ Fig. 2G,H).

\section{Behavioral data in triggered cue experiments}

A slower mean PA was seen across all subjects in the TC trials $\left(7.96 \pm 0.99 \mathrm{~ms}^{-2}\right)$ compared with the RC trials $(9.85 \pm 1.29$ $\mathrm{ms}^{-2}$; two-tailed paired $t$ test; $t_{(9)}=3.19$; $p=0.01)($ Fig. $3 A)$. The mean percentage reduction in PA in TC trials was $19 \pm$ $4.6 \%$ (range, $4-40 \%$ ). Thus, elevations of $\beta$ activity several hundred milliseconds before a motor response exerted a measurable effect during movement execution, with the interval between onset of oscillatory bursts and response being determined by the RT plus the $50 \mathrm{~ms}$ delay imposed by the online $\beta$ filter in these experiments. There was no difference in RT between trial types $(284 \pm 14 \mathrm{~ms}$ in RC trials and $285 \pm 17 \mathrm{~ms}$ in TC trials; two-tailed paired $t$ test; $\left.t_{(9)}=-0.16 ; p=0.87\right)($ Fig. $3 B$ ).

Thus, increased $\beta$ activity was associated with reduced acceleration during voluntary movement. A simple mechanical explanation for this association was discounted by two ancillary experiments. First, we investigated whether phasic increases in $\beta$-frequency band microtremor were associated with changes in the contractile properties of FDI, which thereby slowed movement. To this end, we performed an ancillary experiment in which electrical shocks to FDI were either triggered by $\beta$ microtremor bursts or occurred at random intervals. Finger acceleration driven by direct stimulation of the muscle did not differ, whether shocks were delivered after bursts of $\beta$-band microtremor $\left(14.76 \pm 0.61 \mathrm{~ms}^{-2}\right)$ or at random intervals $(15.15 \pm$ 0.57 ; two-tailed paired $t$ test; $t_{(9)}=1.60 ; p=0.14$ ) (Fig. 4).

We also investigated whether finger acceleration was slowed after TCs because reaction time movements tended to begin during the phase of spontaneous microtremor oscillations involving acceleration in the opposite direction. Accordingly, we subtracted the averaged $\beta$ microtremor signal of the triggered trials from that of the random trials during the period between the trigger oscillation and the sustained acceleration that signaled the start of voluntary movement in each of the 10 healthy subjects. Ninety-five percent confidence limits were calculated for these RC-TC difference traces. Over the $100 \mathrm{~ms}$ before the onset of the reaction time response, three subjects showed net negative accelerations, three subjects showed net positive accelerations, one subject showed both net positive and negative accelerations, and three subjects showed no net accelerations exceeding the $95 \%$ confidence limits. Thus, there was no systematic difference in
B

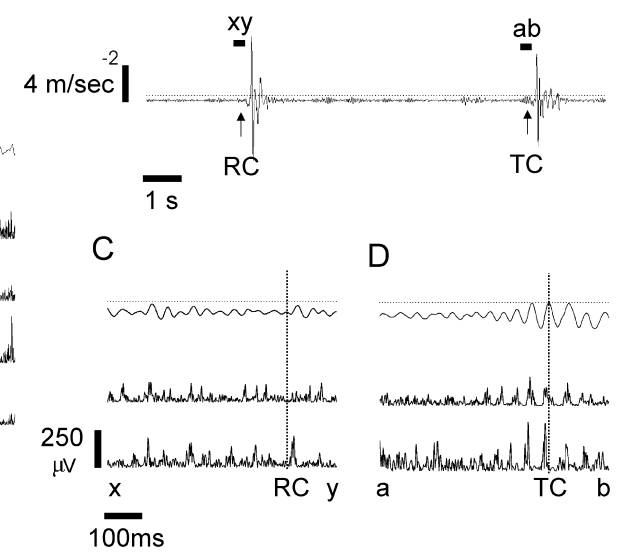

Figure 1. $\beta$-Frequency band microtremor in a single healthy subject. $A$, Spontaneous intertrial microtremor and EMG from ipsilateral and contralateral El and FDI showing lateralized bursts of microtremor (marked by horizontal bars). $\boldsymbol{B}$, Finge movement was made in response to a (thin horizontal lines), with this delay being determined by the on-line electronic $\beta$ bandpass filter. In contrast, before the RC, EMG is desynchronized, and there is little microtremor in the finger. Cue onset is given by the dashed vertical line.

phase locking between trial types, thereby excluding this as a possible cause for slowing in TC trials.

\section{Epidural recordings}

The experiments in healthy subjects indicated that the speed of voluntary finger movements is reduced during periods of elevated microtremor and increased intermuscular synchrony within the $\beta$-frequency band. Both $\beta$-band microtremor of the finger and synchronization between muscles of the distal upper limb are likely to be the product of synchronization of output neurons at the level of the cerebral cortex (McAuley et al., 1997; Halliday et al., 1999; Kilner et al., 1999). Accordingly, we sought direct confirmation that the slowing of finger movements was also associated with elevated $\beta$-frequency band synchrony at the level of the sensorimotor cortex. To this end, we directly recorded the ECoG in two patients (DF and CO) with epidural electrode strips implanted for the treatment of chronic pain by stimulation of the sensorimotor cortex (Fig. 5B). The ECoG, like the microtremor and EMG in healthy subjects, showed phasic bursts of $\beta$ activity lasting several cycles (Fig. $5 A$ ). In the ECoG recorded from contacts 01 in patient DF, bursts of $\beta$ activity occurred on average every $1.4 \mathrm{~s}$ for $1.55 \pm 0.08$ cycles of the PCF, with a maximal duration above $2 \mathrm{SD}$ of 4.4 PCF cycles. ECoG power in the $\beta$ band in contacts 01 was correlated with finger acceleration across RC trials $(n=153)$. Significant negative correlations $(r \geq$ $-0.20 ; p<0.01$ ) existed between wavelet power at frequencies between 18 and $24 \mathrm{~Hz}$ and PA across trials. This was maximal 10 $\mathrm{ms}$ after the cue at $21 \mathrm{~Hz}$, where $r=-0.34$, and $p<0.0001$ (Fig. $5 C, E)$. In patient $C O$, significant negative correlations $(r \geq$ $-0.21 ; p<0.01$ ) were observed at frequencies between 13 and 18 $\mathrm{Hz}$ in contacts 01 overlying the sensory cortex. The maximal correlation occurred $120 \mathrm{~ms}$ before the cue at $15 \mathrm{~Hz}$, where $r=$ -0.44 , and $p<0.0001$ (Fig. $5 D, F$ ). At the PCF in this patient, oscillatory bursts at $15 \mathrm{~Hz}$ in contacts 01 occurred on average every $0.8 \mathrm{~s}$ for $1.89 \pm 0.25$ cycles with a maximal duration of 5.6 cycles. Thus, in both patients, there was a correlation between 
A

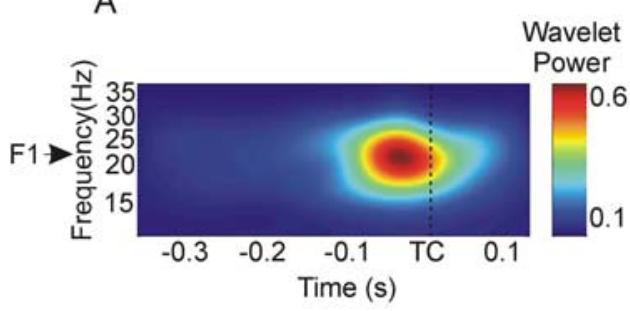

C

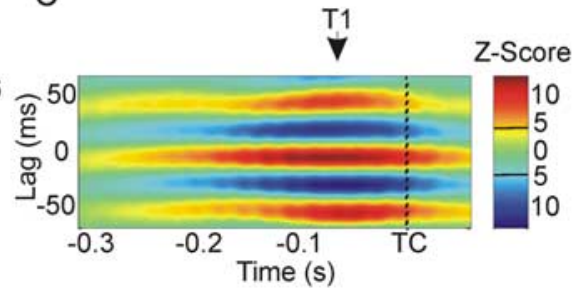

$\mathrm{E}$

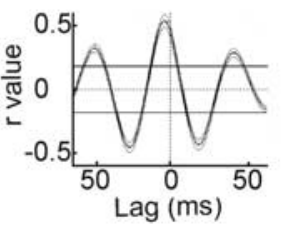

G

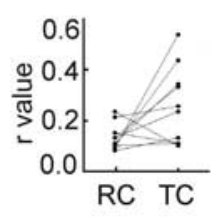

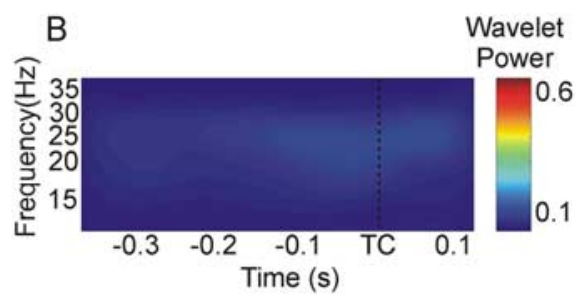

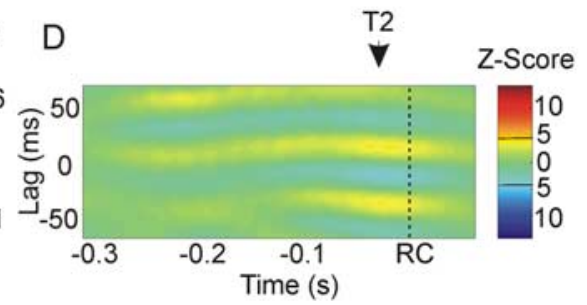

$\mathrm{F}$

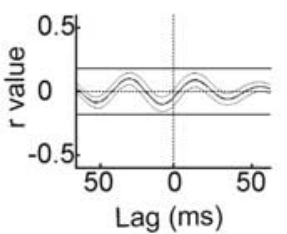

$\mathrm{H}$

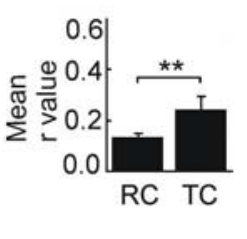

Figure 2. Intermuscular synchrony in a single healthy subject. PA was $18 \%$ less, on average, when cues were triggered ( $n=146$ movements) rather than randomly $(n=72)$ presented. $\boldsymbol{A}, \boldsymbol{B}$, Wavelet transformed microtremor power averaged around TCs and RCS, respectively. C, D, Averaged, time-evolved cross-correlation function between ipsilateral FDI and El EMG at PTF (21 Hz; F1) in TC and RC trials. $\boldsymbol{E}, \boldsymbol{F}$, Two-dimensional cross-correlations (thick lines) at maxima in $($ and $\boldsymbol{D}$ at T1 and T2, respectively, with respective SEMs (thin lines). Ninety-five percent confidence limits are illustrated by the black horizontal lines on both the color plot scales and the two-dimensional correlations. Correlations with a Z score of $>4.75$ were considered significant [level determined after correction for multiple comparisons (129 lag points, 384 time points) by Bonferroni's method] [for previous use of this technique, see Roelfsema et al. (1997)]. This significance level was further verified using a shift control correlation in which no correlations were above this level. $\mathbf{G}, \boldsymbol{H}$, Individual and mean \pm SEM peak intermuscular synchrony during periods of EMG activity before RC and $\mathrm{TC}$ presentation. Error bars represent SEM. ${ }^{* *} p=0.04$ (Wilcoxon signed ranks test).

A

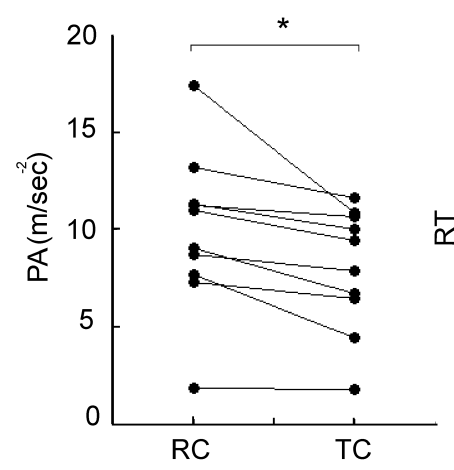

B

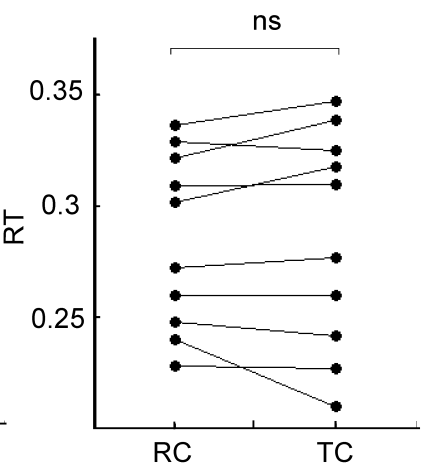

Figure 3. Behavioral data for both TCs and RCs in 10 healthy subjects. $A$, Individual mean PA. ${ }^{*} p=0.01 . \boldsymbol{B}$, Individual mean RT. ns, Not significant $(p=0.74)$.

wavelet power within the $\beta$ band and PA. There was no correlation between wavelet power and reaction time (Fig. $5 G, H$ ).

\section{Differences between trials in triggered stretch experiments}

Subsequently, we investigated the effect of bursts of microtremor in the $\beta$-frequency band on the transcortical stretch reflex. Exaggeration of this response would confirm that microtremor bursts are accompanied by an altered cortical state in healthy subjects and suggest that this cortical state helps promote existing postural set. In this experiment, we simply replaced the visual cue used above with an adducting stretch of the index finger. TSs, but not RSs, followed the onset of bursts of microtremor in the $\beta$-frequency band, and, as expected, bursts of triggering $\beta$ microtremor were unilateral (Fig. $6 A$ ). With only the $50 \mathrm{~ms}$ output delay imposed by the on-line electronic $\beta$ filter, there was no difference between TS and RS in the short-latency spinal M1 component (TS, $0.5 \pm 3 \mu \mathrm{V}$; RS, $4 \pm 2 \mu \mathrm{V}$; two-tailed paired $t$ test; $\left.t_{(9)}=-1.04 ; p=0.326\right)$ or the late $\mathrm{M} 3$ component of the response to stretch (TS, $34 \pm 8 \mu \mathrm{V}$; RS, $26 \pm 6 \mu \mathrm{V} ; t_{(9)}=1.58$;

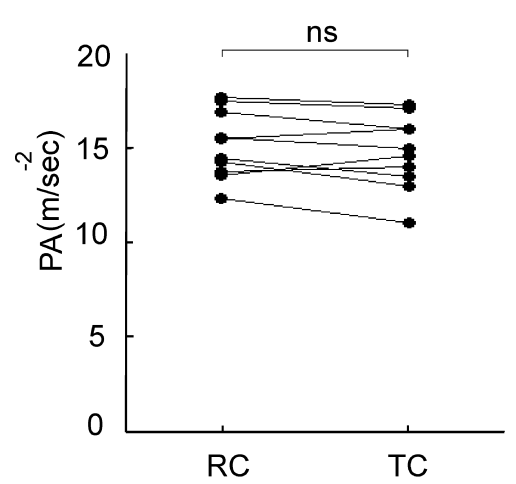

Figure 4. Response to direct electrical stimulation of FDI. Shocks were either delivered triggered (TC) by bursts of $\beta$-frequency band microtremor or presented randomly (RC) in 10 healthy subjects. Individual mean PA in elicited twitches. ns, Not significant ( $p=0.14)$.

$p=0.148)$. In contrast, the transcortical M2 component was significantly higher after TS $(78 \pm 13 \mu \mathrm{V})$ than RS $(62 \pm 10 \mu \mathrm{V}$; $t_{(9)}=4.03 ; p=0.003$ ) (Fig. $\left.6 B, C\right)$.

The exaggeration of the M2 response to stretches delivered after the onset of bursts of $\beta$ microtremor was, however, time limited. The M2 TS/RS ratio was reduced from $1.22 \pm 0.05$ with the 50 ms delay to $1.09 \pm 0.07\left(t_{(9)}=3.459 ; p=0.007\right)$ with a total delay of $250 \mathrm{~ms}$. The ratio after the standard $50 \mathrm{~ms}$, but not the $250 \mathrm{~ms}$, delay differed from unity ( $50 \mathrm{~ms}$ delay, $z=-2.701$, $p=0.007 ; 250 \mathrm{~ms}$ delay, $z=-1.274, p=0.203$; Wilcoxon rank-sum test).

\section{Discussion}

The principal finding is that intrinsic fluctuations in the degree of $\beta$-band synchronization within the motor system are associated with a measurable impairment in movement performance in healthy humans. Specifically, the current results suggest that elevated oscillatory synchrony at the level of the motor cortex may impair local motor processing related to new movements, result- 
ing in decreased force generation and hence response acceleration. This interpretation rests on the assumption that transient changes in the cortical drive to the muscles controlling the index finger are reflected in the nature of the microtremor recorded from the finger with an accelerometer. To what extent is this justified considering the potential confounds of mechanical and spinal influences that might occur to an oscillatory signal as it descends from cortex to the muscles controlling the finger, before being transduced into microtremor through the synchronous activation of motor units? Previous studies have indicated that finger microtremor in the $\beta$-frequency band is attributable to centrally determined synchronization between motor units (McCauley et al., 1997; Halliday et al., 1999), which, in turn, is attributable to synchronization between motor cortex neurons projecting to the spinal cord, as demonstrated in both nonhuman primates (Murthy and Fetz, 1992, 1996; Sanes and Donoghue, 1993; Baker et al., 1997) and humans (Conway et al., 1995; Salenius et al., 1997; Halliday et al., 1999; Kilner et al., 1999; Gross et al., 2000; Mima et al., 2000).

Nevertheless, the assumption that $\beta$ activity in the peripheral motor system reflects synchronization in the same frequency band in the motor areas of the cerebral cortex is so critical to the interpretation of our data that we sought experimental support in three ways. First, we confirmed that periods of elevated $\beta$-frequency band microtremor were accompanied by increased synchronization between motor units in the same band by demonstrating a simultaneous increase in intermuscular synchronization. This kind of rhythmic synchronization between agonist muscles is also thought to be driven by synchronization between motor cortical output neurons (Kilner et al., 2000). Second, we demonstrated that phasic increases in $\beta$-band microtremor were accompanied by a change in the functional state of the sensorimotor cortex as evidenced by an increase in the M2, but not spinal M1, component of the FDI stretch reflex. There is considerable evidence that the M2 stretch response in the hand involves a transcortical loop relayed through primary sensory and then primary motor cortices (Marsden et al., 1976, 1977a,b; Day et al., 1991; Palmer and Ashby, 1992; Macefield et al., 1996; Wallace and Miles, 1998, 2001). Moreover, the failure of the M1 component of the stretch reflex to change suggests that phasic increases in $\beta$-band microtremor are associated with little in the way of alteration of local spinal reflexes. Third, we showed that spontaneous increases in the $\beta$-frequency band component of activity directly recorded from the sensorimotor cortex reduced finger acceleration during our reaction time task. Although this recording was necessarily made after functional neurosurgery in patients with neurological
B

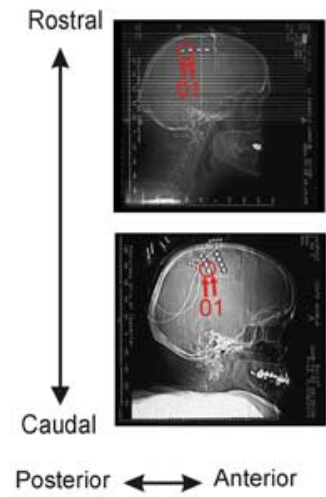

G

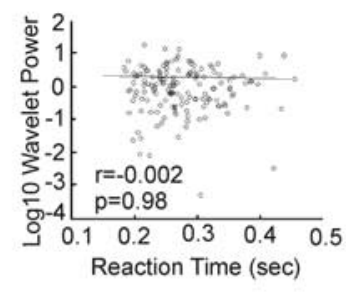

$\mathrm{H}$

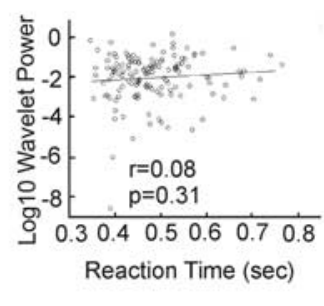

Figure 5. Relationship between $\mathrm{ECOG}$ over sensorimotor cortex and contralateral movement execution in two patients treated for chronic pain. $A$, Example of spontaneous $\beta$ oscillations recorded from patient $D F$, which demonstrates their focal as indicated by arrow b or diffuse character where oscillations are present across all contacts, as in a and c. B, Postoperative skull x-rays from time of a randomly presented cue and Pearson's correlation estimated between ECoG $\beta$-frequency band power at each time point 作 Forrelation at T2, F2 (15 Hz), between log 10 wavelet power, and PA. G, Correlation at T1, F1 (21 Hz), between log 10 wavelet power and RT. $\boldsymbol{H}$, Correlation at T2, F2 (15 Hz), between $\log 10$ wavelet power and RT. The average reaction time was $275 \pm 3 \mathrm{~ms}$ for patient DF and $483 \pm 7 \mathrm{~ms}$ in patient $\mathrm{C} 0$.

disease, the correspondence between the relationships observed in these patients and that determined using our surrogate measures of cortical synchrony in healthy subjects, $\beta$-band microtremor, and intermuscular synchronization was remarkable.

However, several alternative explanations for the apparent association of increased $\beta$ activity with slowed movements should also be considered. First, could phasic increases in $\beta$ microtremor be associated with changes in the contractile properties of FDI and/or fatigue that thereby slow movement? This seems unlikely in the face of the direct evidence of a cortical effect provided by the exaggeration of the M2, but not M1, EMG component of the stretch reflex. This possibility was further refuted by the ancillary experiment in which muscle twitches were compared after triggered and random electrical shocks to the motor point of the FDI. Finger acceleration driven by direct stimulation of the muscle did not differ, whether shocks were delivered triggered by bursts of 

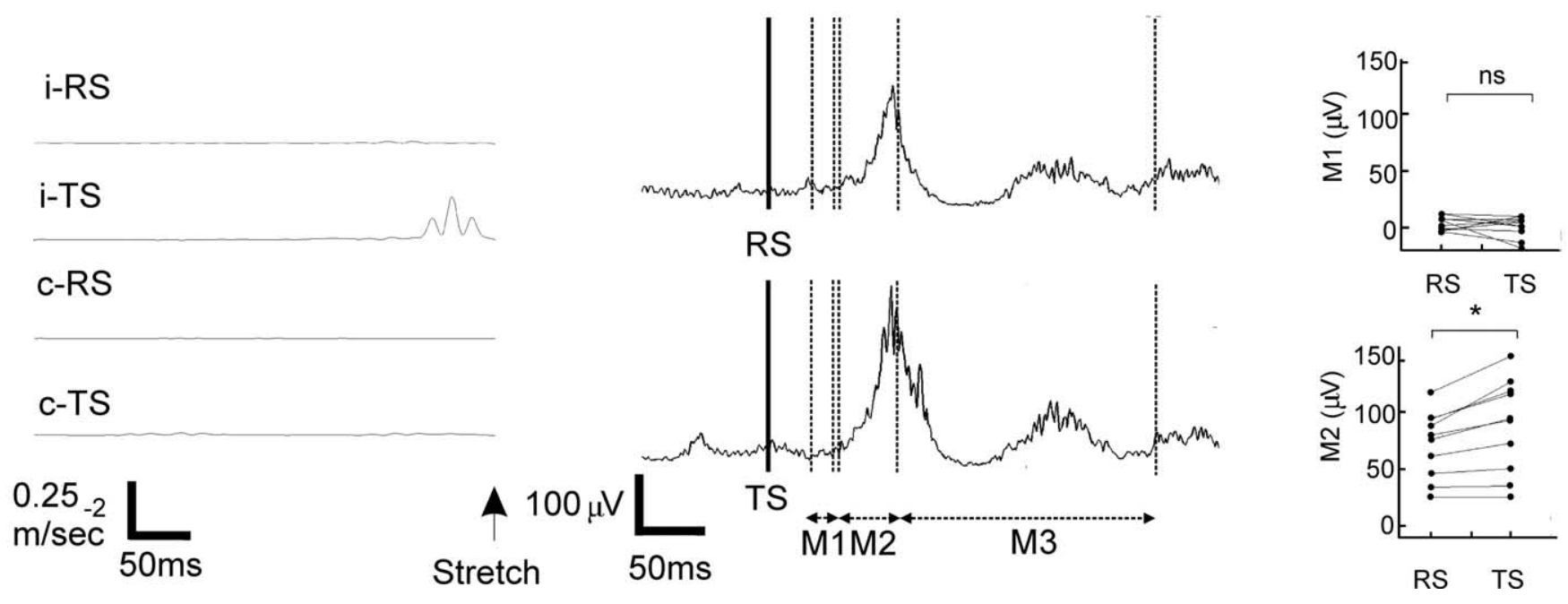

Figure 6. TS experiments. $A$, Average across all subjects of rectified $\beta$ bandpass-filtered acceleration recorded with the ipsilateral (i) or contralateral (c) accelerometer during the $500 \mathrm{~ms}$ before RS or TS. Note the clear transient ipsilateral increase in the $\beta$-band microtremor before the TS. $\boldsymbol{B}$, Averaged EMG responses from a single participant elicited by RS ( $n=50$ trials; top) and TS ( $n=$ 34 trials; bottom). Stretch onset is given by the thick vertical line. Stretch responses were divided into three components: M1 (32-50 ms), M2 (54-100 ms), and M3 (100 - 300 ms). Note that the transcortical $M 2$ response was increased after TS compared with RS, whereas spinal $M 1$ and late $M 3$ responses remained unchanged. $C, M 1$ and $M 2$ responses (mean EMG minus background activity) for both TS and RS in 10 healthy participants. ns, Not significant $(p=0.326) .{ }^{*} p=0.003$; two-tailed paired $t$ tests.

$\beta$-band microtremor or at random intervals. Second, was finger acceleration slowed after increased $\beta$ activity because reaction time movements tended to begin during the phase of spontaneous microtremor oscillations involving acceleration in the opposite direction? However, averages of finger acceleration with respect to movement onset did not show any systematic difference in phase locking between RC and TC trials. Third, could variations in the attention devoted to the task account for both fluctuations in behavioral performance and $\beta$ activity? This again seems unlikely: focused motor attention has been associated with increased rather than reduced $\beta$ synchrony (Kristeva-Feige et al., 2002), and heightened attention would not be expected to reduce the acceleration of motor responses. Indeed, any systematic fluctuation in attention would be anticipated to affect reaction times, yet this parameter did not change in RC and TC trials. Fourth, could small postural changes in the finger confound the results by spuriously triggering visual cues and finger stretches? Subjects were instructed not to make any movements except to the visual cue and care was taken to exclude from the analysis sudden biphasic bursts of large-amplitude acceleration during tonic extension. Furthermore, spuriously triggered cue presentation during a movement would be expected to result in a prolonged reaction time for a second movement in TC trials, whereas stretch reflexes delivered during movement are associated with decreases rather than increases in the long-latency (M2) stretch reflex (Wallace and Miles, 1998, 2001).

The major finding reported here is the slowing of movement made in the setting of spontaneous increases in $\beta$-band synchronization at the level of the sensorimotor cortex. The slowing of movements is not an unexpected feature of alterations in cortical state, because the motor cortex is particularly involved in the coding of force (Maier et al., 1993). The timing of this effect, however, deserves more comment. In primates making reaction time arm movements to visual cues, the earliest neurons to discharge in primary motor cortex do so $140-120 \mathrm{~ms}$ before movement (Evarts, 1972), with intracellular recordings showing slowly rising postsynaptic potentials not $>180 \mathrm{~ms}$ before movement (Matsumura, 1979). Given that the mean reaction time among healthy subjects in our task was $285 \mathrm{~ms}$, elevated corticospinal synchrony and/or its effects would have had to last, at the very least, $105 \mathrm{~ms}$ beyond cue onset to overlap with and influence cortical processing related to the finger movement. However, even estimates of the maximum duration of $\beta$ microtremor bursts fell short of this target. Given a mean PTF of $25 \mathrm{~Hz}$ and the mean maximum duration of $\beta$ microtremor bursts of 3.4 PTF cycles, elevated synchrony, as detected in the periphery, lasted up to $135 \mathrm{~ms}$ after triggering, i.e., no more than $\sim 85 \mathrm{~ms}$ after the cue. Given corticospinal conduction delays of $\sim 20 \mathrm{~ms}$ to FDI (Eisen and Shtybel, 1990), this would imply that bursts of cortical synchrony did not last $>65 \mathrm{~ms}$ after the cue. The results in our patients with epidural cortical recordings were consistent with this, because the peak negative correlation between $\beta$ synchrony at the cortical level and response acceleration occurred around the cue in one patient and $\sim 100 \mathrm{~ms}$ before the cue in the other patient.

The implication is that the phasic bursts of $\beta$ synchrony associated with reductions in the peak acceleration of reaction time responses may precede the onset of the motor cortical activity responsible for such movements. If so, elevated $\beta$ synchrony cannot be an epiphenomenon of any change in the cortical processing related to the slowing of movements but might herald a cortical state, albeit temporary, in which any processing of new movements is impaired. The simultaneous upregulation of the cortical response to stretches after bursts of $\beta$ synchrony suggests that such a cortical state is complex, associated with selective changes in the gain of different cortical circuits according to whether they relate to the processing of new movements or the maintenance of existing tonic activity. Whether changes in gain relate to oscillation-induced modulations of short-term synaptic plasticity is unclear, but the net effect of the elevation in $\beta$ synchrony at the level of the motor cortex is the maintenance of a 
steady-state force output (Schoffelen et al., 2005) and the temporary favoring of the existing motor set.

Synchronization in the $\beta$ range is a characteristic of many levels of the primate motor system raising the question whether such oscillatory activity across neurons has a generic role in promoting the status quo at the expense of novel motor processing, in opposition to the role posited for $\gamma$ synchrony in the motor cortex (Schoffelen et al., 2005). The common suppression of $\beta$ activity before voluntary movement in cortical (Pfurtscheller, 1981), basal ganglia (Cassidy et al., 2002; Levy et al., 2002), and cerebellar (Paradiso et al., 2004) systems would be consistent with such a notion, as would be the emerging evidence that excessive $\beta$ synchrony at cortical and subcortical levels is associated with impaired motor performance in Parkinson's disease (Kühn et al., 2004; Silberstein et al., 2005; Williams et al., 2005).

We have shown that $\beta$-frequency band synchronization within the corticospinal system is associated with measurable effects on movement in healthy subjects. We suggest that $\beta$ synchrony underpins a cortical state in which the existing motor set is favored, and processing related to new movements is penalized.

\section{References}

Baker SN, Olivier E, Lemon RN (1997) Coherent oscillations in monkey motor cortex and hand muscle EMG show task-dependent modulation. J Physiol (Lond) 501:225-241.

Baker SN, Pinches EM, Lemon RN (2003) Synchronization in monkey motor cortex during a precision grip task. II. effect of oscillatory activity on corticospinal output. J Neurophysiol 89:1941-1953.

Brown P, Oliviero A, Mazzone P, Insola A, Tonali P, Di Lazzaro V (2001) Dopamine dependency of oscillations between subthalamic nucleus and pallidum in Parkinson's disease. J Neurosci 21:1033-1038.

Cassidy M, Mazzone P, Oliviero A, Insola A, Tonali P, Di Lazzaro V, Brown P (2002) Movement-related changes in synchronization in the human basal ganglia. Brain 125:1235-1246.

Conway BA, Halliday DM, Farmer SF, Shahani U, Maas P, Weir AI, Rosenberg JR (1995) Synchronization between motor cortex and spinal motoneuronal pool during the performance of a maintained motor task in man. J Physiol (Lond) 489:917-924.

Courtemanche R, Fujii N, Graybiel AM (2004) Synchronous, focally modulated $\beta$-band oscillations characterize local field potential activity in the striatum of awake behaving monkeys. J Neurosci 23:11741-11752.

Day BL, Riescher H, Struppler A, Rothwell JC, Marsden CD (1991) Changes in the response to magnetic and electrical stimulation of the motor cortex following muscle stretch in man. J Physiol 433:41-57.

Donoghue JP, Sanes JN, Hatsopoulos NG, Gaal G (1998) Neural discharge and local field potential oscillations in primate motor cortex during voluntary movements. J Neurophysiol 79:159-173.

Eisen A, Shtybel W (1990) Clinical experience with transcranial magnetic stimulation. Muscle Nerve 13:995-1011.

Evarts EV (1972) Pre- and postcentral neuronal discharge in relation to learned movement. In: Corticothalamic projections and sensorimotor activities (Frigyesi T, Rinvik E, Yaher MD, eds), pp 449-460. New York: Raven.

Farmer SF, Bremner FD, Halliday DM, Rosenberg JR, Stephens JA (1993) The frequency content of common synaptic inputs to motoneurones studied during voluntary isometric contraction in man. J Physiol (Lond) 470:127-155.

Gibbs J, Harrison LM, Stephens JA (1995) Organization of inputs to motoneurone pools in man. J Physiol (Lond) 485:245-256.

Gross J, Tass PA, Salenius S, Hari R, Freund HJ, Schnitzler A (2000) Cortico-muscular synchronization during isometric muscle contraction in humans as revealed by magnetoencephalography. J Physiol (Lond) 527:623-631.

Halliday DM, Conway BA, Farmer SF, Rosenberg JR (1999) Loadindependent contributions from motor-unit synchronization to human physiological tremor. J Neurophysiol 82:664-675.

Kilner JM, Baker SN, Salenius S, Jousmaki V, Hari R, Lemon RN (1999) Task-dependent modulation of $15-30 \mathrm{~Hz}$ coherence between rectified
EMGs from human hand and forearm muscles. J Physiol (Lond) 516:559-570.

Kilner JM, Baker SN, Salenius S, Hari R, Lemon RN (2000) Human cortical muscle coherence is directly related to specific motor parameters. J Neurosci 20:8838-8845.

Kristeva-Feige R, Fritsch C, Timmer J, Lucking CH (2002) Effects of attention and precision of exerted force on beta range EEG-EMG synchronization during a maintained motor contraction task. Clin Neurophysiol 113:124-131.

Kühn AA, Williams D, Kupsch A, Limousin P, Hariz M, Schneider GH, Yarrow K, Brown P (2004) Event-related beta desynchronization in human subthalamic nucleus correlates with motor performance. Brain 127:735-746.

Kühn AA, Trottenberg T, Kivi A, Kupsch A, Schneider G-H, Brown P (2005) The relationship between local field potential and neuronal discharge in the subthalamic nucleus of patients with Parkinson's disease. Exp Neurol 194:212-220.

Levy R, Ashby P, Hutchison WD, Lang AE, Lozano AM, Dostrovsky JO (2002) Dependence of subthalamic nucleus oscillations on movement and dopamine in Parkinson's disease. Brain 125:1196-1209.

Macefield VG, Rothwell JC, Day BL (1996) The contribution of transcortical pathways to long-latency stretch and tactile reflexes in human hand muscles. Exp Brain Res 108:147-154.

Maier MA, Bennett KM, Hepp-Reymond MC, Lemon RN (1993) Contribution of the monkey corticomotoneuronal system to the control of force in precision grip. J Neurophysiol 69:772-785.

Marsden CD, Merton PA, Morton HB (1976) Stretch reflex and servo action in a variety of human muscles. J Physiol (Lond) 259:531-560.

Marsden CD, Merton PA, Morton HB, Adam J (1977a) The effect of posterior column lesions on servo responses from the human long thumb flexor. Brain 100:185-200.

Marsden CD, Merton PA, Morton HB, Adam J (1977b) The effect of lesions of the sensorimotor cortex and the capsular pathways on servo responses from the human long thumb flexor. Brain 100:503-526.

Marsden CD, Rothwell JC, Day BL (1983) Long-latency automatic responses to muscle stretch in man: origin and function. Adv Neurol 39:509-539.

Marsden JF, Ashby P, Limousin-Dowsey P, Rothwell JC, Brown P (2000) Coherence between cerebellar thalamus, cortex and muscle in man: cerebellar thalamus interactions. Brain 123:1459-1470.

Matsumura M (1979) Intracellular synaptic potentials of primate motor cortex neurons during voluntary movement. Brain Res 163:33-48.

McAuley JH, Rothwell JC, Marsden CD (1997) Frequency peaks of tremor, muscle vibration and electromyographic activity at $10 \mathrm{~Hz}, 20 \mathrm{~Hz}$ and 40 $\mathrm{Hz}$ during human finger muscle contraction may reflect rhythmicities of central neural firing. Exp Brain Res 114:525-541.

Mima T, Steger J, Schulman AE, Gerloff C, Hallett M (2000) Electroencephalographic measurement of motor cortex control of muscle activity in humans. Clin Neurophysiol 111:326-337.

Murthy VN, Fetz EE (1992) Coherent 25- to 35-Hz oscillations in the sensorimotor cortex of awake behaving monkeys. Proc Natl Acad Sci USA 89:5670-5674.

Murthy VN, Fetz EE (1996) Synchronization of neurons during local field potential oscillations in sensorimotor cortex of awake monkeys. J Neurophysiol 76:3968-3982.

Palmer E, Ashby P (1992) Evidence that a long latency stretch reflex in humans is transcortical. J Physiol (Lond) 449:429-440.

Paradiso G, Cunic D, Saint-Cyr JA, Hoque T, Lozano AM, Lang AE, Chen R, $\mathrm{G}$ (2004) Involvement of human thalamus in the preparation of selfpaced movement. Brain 127:2717-2731.

Pfurtscheller G (1981) Central beta rhythm during sensorimotor activities in man. Electroencephalogr Clin Neurophysiol 51:253-264.

Roelfsema PR, Engel AK, Konig P, Singer W (1997) Visuomotor integration is associated with zero time-lag synchronization among cortical areas. Nature 385:157-161.

Salenius S, Portin K, Kajola M, Salmelin R, Hari R (1997) Cortical control of human motoneuron firing during isometric contraction. J Neurophysiol 77:3401-3405.

Samar VJ, Bopardikar A, Rao R, Swartz K (1999) Wavelet analysis of neuroelectric waveforms: a conceptual tutorial. Brain Lang 66:7-60.

Sanes JN, Donoghue JP (1993) Oscillations in local field potentials of the 
primate motor cortex during voluntary movement. Proc Natl Acad Sci USA 90:4470-4474.

Schoffelen JM, Oostenveld R, Fries P (2005) Neuronal coherence as a mechanism of effective corticospinal interaction. Science 308:111-113.

Sharott A, Magill PJ, Harnack D, Kupsch A, Meissner W, Brown P (2005) Dopamine depletion increases the power and coherence of beta (15-30 $\mathrm{Hz}$ ) oscillations in the cerebral cortex and subthalamic nucleus of the awake rat. Eur J Neurosci 21:1413-1422.

Silberstein P, Kühn AA, Kupsch A, Trottenberg T, Krauss JK, Wohrle JC, Mazzone P, Insola A, Di Lazzaro V, Oliviero A, Aziz T, Brown P (2003) Patterning of globus pallidus local field potentials differs between Parkinson's disease and dystonia. Brain 126:2597-2608.

Silberstein P, Pogosyan A, Kuhn AA, Hotton G, Tisch S, Kupsch A, Dowsey-Limousin P, Hariz MI, Brown P (2005) Cortico-cortical coupling in Parkinson's disease and its modulation by therapy. Brain 128:1277-1291.

Strambi SK, Rossi B, De Michele G, Sello S (2004) Effect of medication in
Parkinson's disease: a wavelet analysis of EMG signals. Med Eng Phys 26:279-290.

Torrance C, Compo GP (1998) A practical guide to wavelet analysis. Bull Am Meteorol Soc 79:61-78.

Wallace CJ, Miles TS (1998) Movements modulate the reflex responses of human flexor pollicis longus to stretch. Exp Brain Res 118:105-110.

Wallace CJ, Miles TS (2001) Cortical excitability is not depressed in movement-modulated stretch response of human thumb flexor. Exp Brain Res 139:448-453.

Williams D, Tijssen M, Van Bruggen G, Bosch A, Insola A, Di Lazzaro V, Mazzone P, Oliviero A, Quartarone A, Speelman H, Brown P (2002) Dopamine-dependent changes in the functional connectivity between basal ganglia and cerebral cortex in humans. Brain 125:1558-1569.

Williams D, Kühn AA, Kupsch A, Limousin P, Hariz M, Schneider GH, Yarrow K, Brown P (2005) The relationship between oscillatory activity and motor reaction time in the parkinsonian subthalamic nucleus. Eur J Neurosci 21:249-258. 